Fig. 4. Die tieferen Schichten der Hornhaut, unter dem Hornhautgeschwür, mit Nestern von Friedländer-Bacillen. Methylenblaufärbung. Zeiss apochrom. $2 \mathrm{~m} / \mathrm{m}$. Ocul. $\mathrm{A}$.

Fig. 5. Friedländer-Bacillen im Glaskörper nach Einimpfung (Panophthalmie). Löffler's Methylenblau.

Fig. 6. Friedländer'sche Bacillen in der Netzhaut, nach Einimpfung derselben in den Glaskörper. Eosin-Metbylenblaufärbung. Zeiss apochrom. Immers. $2 \mathrm{~m} / \mathrm{m}$. Ocul. II. $5 \mathrm{~cm}$ ab Boden.

Fig. 7. Linsenabscess nach Impfung von Diphtberiebacillen in die Vorderkammer.

Fig. 8. Cyclitis und perilenticulärer Glaskörperabscess, nach Impfung von Fränkel'schen Diplokokken in den Glaskörper.

Fig. 9. Retinitis nach Impfung Fränkel'scher Kokken in den Glaskörper. Kokken auch in der Retina. Färbung nach Gram.

Fig. 10. Hornbautgeschwür mit Fränkel'schen Kokken. Hämatoxylin-Eosinfärbung.

Fig.11. Fränkel'sche Kokken in der Hornhaut. Färbung nach Gram.

Näheres zur Erläuterung der Figuren ergiebt der Text.

\title{
XI.
}

\section{Ueber Corpora amylacea und locales Amyloid in einem endostalen Sarcom des Brustbeins.}

(Aus der Göttinger chirurgischen Klinik.)

\author{
Von Professor 0. Hildebrand.
}

(Hierzu Taf. VII.)

Als ich bei meinen Untersuchungen über die Anwesenheit von Glykogen in Geschwülsten ${ }^{1}$ ) die Sammlung der chirurgischen Klinik auf Tumoren durohmusterte, die einen derartigen Befund vermuthen liessen, stiess ich auf ein schon seit langen Jahren aufbewahrtes Präparat, das bei der mikroskopischen Durchforschung ein recht eigenthümliches Bild darbot. Es war ein Tumor des Sternum, den Prof. König im Jahre 1882 durch Operation entfernte. Die Geschichte der Operation wurde damals auf der Naturforscherversammlung in Eisenach von

1) Langen beck's Archiv. Bd. 47. 


\section{Prof. König mitgetheilt und im Centralblatt für Chirurgie ${ }^{1}$ ) publicirt.}

Es handelte sich um eine 36 jährige Frau, die seit $2 \frac{1}{2}$ Jahren eine Geschwulst des Brustbeins hatte ohne ansser wechselnden Schmerzen wesentliche Störungen davon zu erleiden. Die Frau war sonst gesund, es liess sich nichts Krankes weiter an ihr nachweisen. Die Geschwulst erstreckte sich auf das Corpus sterni, überragte dasselbe nach oben, hier noch etwas über die Insertionsgrenze der II. Rippe hinausgebend. Seitlich überschritt sie die Brustbeingrenze nur wenig. Sie hatte halbovale, ziemlich gleichmässige Form, war vollkommen unverschieblich und mit zwar verdünnter, aber gesunder Haut überzogen. Die Diagnose wurde auf einen endostalen Tumor des Brustbeins gestellt und das Sternum sammt dem Tumor resecirt. Die dabei eingetretene Eröffnung aller Hohlräume der Brust überstand Pat. gut. Sie wurde geheilt. 1 Jahr und 2 Monate später starb Pat. nach langdanerndem Muskelrheumatismus, der sie sehr heruntergebracht hatte, und Albuminurie.

Bei der jetzigen Untersuchung des Präparates, das im Alkohol ziemlich stark geschrumpft ist, findet sich Folgendes: Die Geschwulst ist etwa faustgross, von ziemlich gleichmässiger, ovaler Form, stellenweise etwas gebuckelt. Oben und unten ragt aus derselben Knochen hervor, die Enden des Sternum, während zu beiden Seiten die mitentfernten Theile von je 6 Rippen aus der Geschwulst hervorschauen. Eine kaöcherne Schale hat der Tumor nicht. Auf dem Durchschnitt durch die Neubildung erkennt man, dass von dem Brustbein nur noch kleine Reste, beide Enden vorhanden sind, während der ganre mittlere Theil in der Ausdehnung von $8 \mathrm{~cm}$ durcb Geschwulstmasse ersetzt ist und zwar so vollständig, dass man gar keine Spur von Knochen wahrnimmt. Beim Durchschnoiden knirscht der Tumor nicht; dabei ist er nicht entkalkt. Die Schnittfläche der Geschwulst sieht meist homogen, weisslichgrau aus, $a b$ und $2 u$ nimmt man gelbliche Partien, Streifen wahr.

Die mikroskopischen Befunde, die ich bei der Untersuchung der Geschwulst erbob, waren folgende.

Zunächst fällt an den Schnitten auf, dass sie, wenn sie im Wasser schwimmen, stellenweise sehr durchsichtig sind. Unter dem Mikroskop bieten sie ein recht mannichfaltiges Bild, indem sie das Geschwulstgewebe zum Theil in reiner, zum Theil aber auch in sehr modificirter Form zeigen. Der Hauptsache nach besteht das Geschwulstgewebe aus mittelgrossen, rundlichen Zellen, die durch zarte Bindegewebsfasern von einander getrennt sind. Nur ab und zu vereinigen sich letztere zu derberen Fasern und Bündeln. Da, wo die Zellen ausgefallen sind, sieht man ein enges Gitterwerk, meist für je eine Zelle ein Gitter. Die Zellen sind rundlich, oval, zum Theil aucb polygonal, der Kern ist mittelgross, ebenfalls oval oder rund, oft mit einer Anzahl bei Hämatoxylinfärbung stärker pigmentirter

1) Centralblatt f. Chirurgie. 1882. No. 42 . 
Körner versehen. Zwischen ihnen liegen oft grössere, wie gequollene, ovale Zellen mit viel grösserem, weniger intensiv gefärbtem Kern, deren Zellleib leicht granulirt ist und mit Eosin sich rosa färbt. Bei einzelnen dieser Zellen ist der Kern an die Peripherie gerückt und man hat den Eindruck, dass er dabin durch Quellung der Zelle gelangt sei. Mancbe Zellen haben auch mebrere erbeblich grössere Kerne, die blasser und mit einer Anzahl von kleinen Kernkörperchen versehen sind. Mitten zwischen den Rundzellen des Geschwulstgewebes liegen ab und zu ganz grosse, 10-15 mal so grosse Zellen, also Riesenzellen mit einer grösseren Anzahl von Kernen. Ausserdem bilden noch weite, mit einfachem Endothel ansgekleidete Lumina einen Bestandtheil der Geschwulst, Lumina, an deren Endotbelrohr aussen direct die Geschwulstzellen anstossen und die innen mit Blutkörperchen gefüllt sind. In diesem Gescbwulstgewebe sind nun stellenweise eine Unmenge runder homogener Körper der verschiedensten Grösse sichtbar. Diese liegen theils in grossen Haufen dicht gedrängt bei einander, und bilden grosse Bezirke für sich, theils in geringer Anzabl im übrigen Geschwulstgewebe verstreut, doch aucb manehmal in einer grossen Partie eines Schnittes nur ein solcher Körper. Gewöhnlich sind sie nur durch ganz schmale Bindegewebssepten von einander getrennt, so dass man an das Bild von Fettgewebe erinnert wird, so regelmässig rund sind vielfach die Körper, so schroal sind die zwischen liegenden Bindegewebsbrücken. Oft sind diese Körper kreisrund. Die grösseren zeigen meist eine concentrische Schichtung, wie die Schnittfäche eines geschichteten Steines, eines Blasensteines; an einem konnte ich 7 concentrische Ringe zählen. Dazu kommt nach eine radiäre Streifung, die meist von dem Centrum bis zur Peripherie durchgeht. Diese radiäre Streifung ist sehr fein und zart, aber sehr deutlich. An manchem Körper fand ich diese radiäre Streifung nur im centralen Theil, an anderen war sie ausgesprochen nur in der peripherischen Ringschicht vorhanden; meist ist sie hier am dentlichsten. Gelegentlich erweckt sie bier den Eindruck von zarten Franzen odei Falten. Die kleinen Körperchen, die oft nur die Grösse einer gewöbnlichen Rundzelle haben und stets kreisrund sind, sind nicht concentrisch geschichtet, jedoch meist ist an ihnen eine radiäre Streifung bemerkbar. Die freiliegenden Corpora zeigen nie, weder am Rand noch innen etwas, was wie ein Zellkern aussähe. - $\mathrm{Ab}$ und zu finden sich nun aber auch sebr unregelmässig und verscbieden gestaltete Corpora mit unebener Oberfäche. Oft haben sie länglich ovale Gestalt, gelegentlich im Innern einen undeutlichen centralen Theil oder Spalt. Oft modeln sie ihre Form nach den daneben liegenden. $A b$ und zu nimmt man auch grosse Körper wahr, die bucklig oder gelappt aussehen, als ob sie aus mehreren zusammengeflossen seien, oder auch mächtige Schollen der gleichen homogenen Masse. So liegt an einer Stelle ein Haufen, der noch erkennen lässt, dass er aus 3 solchen Körpern zusammengeflossen ist, deren jeder jedoch seine radiäre Streifung so weit für sich behalten hat, dass man sie an den Auflagerungsstellen noch eine Strecke weit im Innern des Ganzen verfolgen kann (Fig. 3). An einer 
ánderen grossen Scholle, die eine gleichartige peripherische radiäre Streifung hat, erkennt man deutlich, dass sie im Innern mehrere concentrisch geschichtete Kugeln birgt, an welchen man keine radiäre Streifung sieht (Fig. 1). An einer weiteren Stelle liegt eine Riesenscbolle von sehr unregelmässiger Gestalt mit einer exquisiten Streifung der Randzone. Diese Scholle ist im Uebrigen feinkörnig, fein granulirt, nahe dem Rand ist die Substanz homogener als im Centrum, eine ausgesprochene Struktur ist aber nicht vorhanden. Es sind in ihr einige kleine Zellkerne eingeschlossen. Solche Schollen finden sich vielfach. Nicht weit von einer solchen sieht man ein mächtiges Zellconglomerat, ebenfalls von einem homogenen Saum umgeben. In einzelnen der grossen conglomerirten Schollen sieht man deutliche Kerneinschlüsse, die wohl die Reste sind jener zarten Bindegewebsmaschen um die einzelnen Corpora herum. Die gleiche homogene Masse sieht man an verschiedenen Stellen direct um grosse, dünnwandige Gefässe herum gelagert und an einigen dieser Gefässe sieht es fast so aus, als wären diese bomogenen Massen hervorgegangen aus zusammengeflossenen gequollenen Zellen, die direct um das Endotbelrohr herum lagen; man findet nebmlich innerhalb der homogenen Masse radiäre Streifung angedeutet und an der äusseren Grenze dieser homogenen Masse Einkerbungen, die einzelnen dieser Streifen entsprechen, so dass man diese für Zellgrenzen balten könnte. Freilich könnten diese Bilder auch durch Druck auf das Deckgläschen entstanden sein. Oftmals aber liegen zwischen diesem homogenen Ring und dem Endothelrohr noch einige wenige lockere Bindegewebsfasern. Vielfach sind die Gefässe auch ganz nuverändert. In mehreren weiten dünnwandigen Gefässlumina, die mit blassen rotben Blutkörperchen angefüllt sind, finden sich vereinzelte kleine Corpora, die an ihrer radiären Streifung und ibrer Grösse deutlich zu erkennen sind. Sie sind etwa 2-3 mal so gross, als ein weisses Blutkörperchen. Ausserdem finden sich noch ovale Zellen mit deutlich gefärbtem, blassem, rundlichem Kern im Lumen. - Schliesslich durchziehen stellenweise schmale glänzende Bänder, gequollene dicke Fasern und Aeste die Geschwulst, als ob Bindegewebsfibrillen dieselben homogenisirenden Umwandlungen eingegangen wären. - An einer Stelle sitzen um die Körper die Geschwulstzellen in mehreren Reihen so dicht und bilden mit dem eingeschlossenen Corpus jedesmal ein so isolirtes Gewebshäufchen, dass man zu der Vermuthung lrommt, dass erstere aus letzteren hervorgegangen seien.

Färbt man die Schnitte mit Hämatoxylin-Eosin, so werden jene Körper, jene Schollen alle rosigroth, ebenso wie die Leiber der Geschwulstzellen, während die Zellkerne blau gefärbt sind. Dabei verschwindet die radiäre Streifung, die concentrische Schichtung fast ganz. Bei dieser Färbung werden nun auch die grossen, mehr- oder vielkernigen Zellen deutlicher, die zum Theil sehr grosse, fein granulirte Zellleiber haben und meist etwas mehr gelblich-rosa gefärbt sind. In denselben sind die reichlichen Kerne theils wandständig, theils mehr central angeordnet. Oft liegen grössere Zellen dicht neben einem jener homogenen Körper, manchmal 
sitzt dem letzteren eine mondsichelförmige, granulirte grosse Zelle auf, die einen oder mehrere Kerne bat. In einzelnen dieser grossen Zellen nimmt man in dem granulirten Protoplasma bomogene, runde Partien wabr, die zum Theil die eine Hälfte der Zelle einnehmen, während die Kerne in die andere gerückt sind (Fig.9), zum Theil aber auch mehr central liegen, während die Kerne die Peripherie besetzt halten (Fig. 7). - An einer Stelle fand ich auch einen radiär gestreiften Körper, dessen Peripherie verschieden geformte, fein granulirte, kernbaltige Zellen aufsitzen, jedoch so aufsitzen, dass eine Grenze zwischen beiden nicht zu erkennen ist, sondern sie unmittelbar in einander äberzugehen scheinen, indem auch die radiäre Streifung sich in die Zellleiber zu erstrecken scheint. Man hat hier also den Eindruek, als ob die Zellen mit dem Körper verschmolzen seien (Fig. 10).

Bei der Untersuchung der dicht am Knochen gelegenen Geschwulsttheile ergiebt sicb derselbe Befund. Dabei findet man das Geschwulstgewebe zwiscben den Knocbenbälkcben. An einer Stelle sieht man hyalinen Knorpel und dicht daneben Geschwulstgewebe mit vielen Körpern, einzelne der letzteren auch in dem Knorpelbezirk. Ferner bemerkt man rundlicbe ovale Schollen gelbkrümeliger Masse, in denen eine blasse homogene Kugel liegt. Hier kann man nun auch deutlich sehen, dass die zarte Umgrenzungshaut um die Corpora nicht ihnen selbst angebört, sondern dass es Septen sind.

Legt man nun die Schnitte in Jodjodkalilösung, so werden sowohl jene runden Körper, wie die Schollen braunrotb, oder grünlich-gran oder blaugrün, zum Theil bekommen sie aber auch nur einen schmutzig-grünen oder grünblauen Ring aussen am Rand. Manchmal ist auch blos das Centrum dunkler, während der äussere umgelagerte Ring viel heller leuchtend jst, ja manchmal wird das Centrum braunrotb, die concentrischen Ringe darum aber blaugrün. Und diese Färbung verscbwindet nicht so rasch, sie ist z. B. viel beständiger, als die Glykogenfärbung. Nach Zusatz von Schwefelsäure zu diesen Jodpräparaten werden sie zu einem Theil viel intensiver braunroth, glänzend braunrotb, meist jedoch violett, chocoladefarben, blaugrün, blau, ja blauschwarz, wobei gewöbnlich ein rundes oder ovales Centrum dunkler gefärbt ist. Die concentrische Schichtung bleibt sehr deutlich; zwischen den einzelnen concentrischen dunklen Ringen siebt man oft eine schmale hellere, glänzende Zone. Auch die radiäre Streifung ist an den Jodpräparaten sehr deutlich; man kann dann vielfach genau sehen, dass sie vom Centrum zur Peripherie reicht. Sebr schön wurde die Färbung, wenn die Schnitte vor der Jodfärbung in verdünnter Schwefelsäure gelegen hatten, namentlich die concentrische Schichtung wurde dann sehr deutlich. Am ausgesprochensten aber trat die Blaufärbung jener Schollen u. s.w. in Gegensatz zu der Blassgelbfärbung des übrigen Gewebes hervor, wenn die Schnitte nach der Jodfärbung tagelang in dünner Schwefelsäure gelegen hatten. Es gab dann eine sehr distincte Fürbung. Durch diese Färbung sieht man auch Corpora, die kleiner sind, als die Zellen. Von einem Kerne 
im Sinne Friedreich's ${ }^{1}$, Langhans' ${ }^{2}$ ) ist in meinem Fall nichts wahrzunehmen. Ausser jenen runden Körpern nebmen dieselbe blaugrüne Farbe auch jene ungleichen Schollen und auch manche Gewebspartien an, in denen man die Zellen nur noch sehr undeutlich siebt. Ausserdem treten aber im Bindegewebe eine Menge Züge, Balken, Aeste u. s. w. ebenfalls in bläulicher Farbe hervor, jedoch weniger intensiv, während das übrige Gewebe namentlich gewöhnliches Bindegewebe gelb, quergestreifte Muskeln intensiv gelb erscheinen. Mit Methylviolett werden die Corpora leuchtend dunkelroth, das Gewebe blau, mit Pikrocarmin die Corpora gelb, das Gewebe, roth, mit Modeb aun die Corpora hellbraun, die Zellkerne intensir braun, dabei ist die Schichtung noch sehr deutlich. Saffranin färbt die Corpora graugelb, Jodgrün macht sie blassgrün, das Gewebe intensiv grün, Gentianaviolett roth, das Gewebe blau, Eosin roth und etwas homogener, die Schichtung wird undeutlicher. Weigert'sche Fibrinfärbung färbt die Corpora blassgraublau, ja gelblich, während die Kerne der Geschwulst noch intensiv blau gefärbt sind. Werden die Scbnitte nach Ernst mit van Gieson'schem Gemisch gefärbt, so werden sowobl die Geschwulstzellen, als die Corpora gelb, die Knochenbalken jedoch granatroth; doch werden die Corpora rothgelb, während die Zellen graugelb aussehen, ebenso wie die Riesenzellen. Essigsäure, Salpetersäure, Schwefelsäure, Salzsäure in dünnen Lösungen sind ohne wesentlichen Einfluss, bei stärkeren Lösungen dieser Säuren werden die Gorpora durchscheinender, heller und die Schichtung wird undeutlicher. Concentrirte Schwefelsäure löst sie in wenigen Augenblicken. Kochende Salpetersäure ebenfalls vollständig.

Alkalien haben keinen Einfluss.

Bei Bebandlung mit Osmiumsäure bleiben die Corpora blass.

Aether löst sie nicht, eben so wenig hat Chloroform in dieser Richtung einen Einfluss. An letzteren Präparaten, die sehr aufgehellt sind, sieht man bei der Untersuchung in Glycerin noch sehr gut die concentrische und radiäre Streifung. Auch kochender Alkoboläther löst sie nicht, die Jodfärbung gelingt eben so gut wie vorher, die Schichtung bleibt gut sichtbar.

Kochendes Wasser löst die Corpora nicht, sie quellen auch nicht auf, wie Virchow ${ }^{3}$ ) an seinen Präparaten gefunden hatte; es lässt sich dann noch sehr gut die Jod- und Jodschwefelsäurereaction zur Anschauung bringen.

Zusatz von Speichel zu den Schnitten hatte gar keinen Einfluss. Bei Auslaugung eines zerkleinerten Theiles des Präparates mit Wasser und nachfolgender Speichelbehandinng lässt sich kein Zucker nachweisen mit Hülfe der Trommer'schen Probe.

1) Dieses Archiv. Bd. 9.

2) Dieses Archiv. Bd. 38.

3) Dieses Archiv. Bd. 8 . 
Es gelang mir eben so wenig, wie Virchow ${ }^{1}$ ) und Donders ${ }^{2}$ ) die Beobachtung Busk's ${ }^{3}$ ) zu machen, dass bei der Betrachtung der Corpora im polarisirten Lichte ein Kreuz zu sehen sei u. s. w.

Alle diese Reactionen zeigen die Corpora, die unregelmässigen Schollen, die homogenen Ringe um die Gefässe, die homogenen Aeste und Züge in ganz gleicher Weise. An manchen Stellen, da wo die Corpora nicht so dicht liegen, sondern zwischen ihnen Geschwulstzellen liegen, da haben sie oft als äusserste Schicht einen nach Jodfärbung hellbräunlich glänzenden Ring von Geschwulstzellen, ja sogar mehrere solcher Schichten. Die Abgrenzung der Zellen gegen einander ist hier dann nicht mehr deutlich (Fig. 2).

Fassen wir nun das Wesentliche dieser Beobachtungen zusammen. Es handelt sich hier also um ein grosses endostales Rundzellensarcom des Sternum, in welchem an manchen Stellen in grosser Zahl rundliche, blasse, glänzende Körper von sehr verschiedener Grösse eingelagert sind, die mehr oder weniger deutlich concentrisch geschichtet und radiär gestreift sind, deren Farbenreaction sich mit der der Corpora amylacea bezw. des Amyloids deckt. Ausser diesen trifft man erstens noch grössere analoge, jedoch unregelmässig geformte Schollen ebenfalls mit radiärer Streifung von gleichem Aussehen und gleicher Reaction, wie jene, die manchmal den Eindruck machen, als wären sie durch Zusammenfliessen mehrerer von jenen runden Körpern entstanden: Forner finden sich noch um einzelne Gefässe herum breite homogene Bänder von gleichem Aussehen und Reaction, jedoch ohne Schichtung und radiäre Streifung und schliesslich Bänder und Streifen homogenen glänzenden Gewebes, die ebenfalls dieselbe Reaction geben.

Der Fall bietet also nach mancherlei Richtung Interesse. Sind jene runden Körper Corpora amylacea? Bekanntlich kommen ächte Corpora amylacea in wirklichen Geschwülsten recht selten vor. Aber vielleicht giebt uns dieser Fall auch etwas Licht über die Beziehung zwischen Corpora amylacea und localer Amyloidablagerung und über die Genese dieser beiden.

Wie sind nun zunächst jene runden Körper aufzufassen? Da verdünnte Lösungen starker Säuren ohne Einfluss auf die-

1) Dieses Archiv. Bd. 8. S, 367.

2) Ebenda.

3) Quarterly Journal of micr. science. 1854. Jan. No.6. 
selben bleiben, so sind etwaige Concretionen anorganischer Salze, wie kohlensaurer Kalk, an den man aus verschiedenen Gründen ja denken könnte, bestimmt ausgeschlossen. Die Aehnlichkeit mit Blasensteinen ist nur eine morphologische. Der Sitz des Tumors aber legt die Vermuthung nahe, dass man es hier vielleicht mit Hassal'schen Körperchen der Thymusdrüse zu thun habe. Dann müsste man den ganzen Fall so auffassen, dass bei der Bildung des Sternums aus den paarigen Rippenknorpelleisten ein Thymustheil in das Sternum verlagert worden sei, dass dieser sich zu einem Rundzellensarcom entwickelt habe und jene bekannten Hassal'schen Körperchen sich in ihm gebildet hätten. Gegen diese ganze Combination spricht freilich, dass jene Körper keine Hassal'sche Körper sein können. Denn diese sind nicht in der regelmässigen Weise wirklich concentrisch geschichtet, wie jene oben beschriebenen Körper, was ja bei ihrer Entstehung aus zusammengeschichteten Zellen ohne Weiteres klar ist; es fehlt ihnen ferner die radiäre Streifung vollständig, und dann geben sie nach Virchow ${ }^{1}$ ) keine Jodschwefelsäurereaction. Also dieser Versuch der Erklärung muss zurückgewiesen werden. - Nach

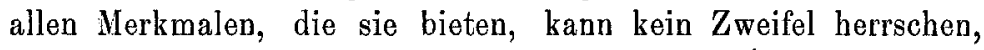
dass sie zu jenen Bildungen gehören, die man als Corpora amylacea bezeichnet. Diese Körper sind ja durch die Untersuchungen Virchow's ${ }^{2}$ ), Friedreich's ${ }^{3}$ ), Zahn's ${ }^{4}$, Langhans's ${ }^{5}$, Paulicki's ${ }^{6}$ ), Jürgens's ${ }^{7}$ ), Stilling's ${ }^{8}$ ), Posner's ${ }^{9}$ ), Favre's ${ }^{10}$ ), Ceci's ${ }^{11}$ ), Siegert's ${ }^{12}$ ) und Anderer hinlänglich bekannt geworden, als häufiger Bestandtheil der Lunge, des Centralorgans des Nervensystems, der Prostata, der Schleimhaut

1) Dieses Archiv. Bd. 6.

2) Dieses Archiv. Bd.6, 8 .

3) Dieses Archiv. Bd. 9, 10, 30.

4) Dieses Archiv. Bd. 72.

5) Dieses Archiv. Bd. 38.

6) Dieses Archiv. Bd. 16.

7 Dieses Archiv. Bd. 65.

8) Dieses Archiv. Bd. 98.

9) Zeitschr. f. klin. Med. 1889. Bd. 16.

10) Thèse de Genève. 1879. Corpuscules de l'appareil urogenital.

11) Reale Academia dei Lincei. 1881. Ser. 39. Vol. IX.

12) Dieses Archiv. Bd. 129. 
der Harnwege. Aber ausser diesen Befunden in normalen oder entzündeten Geweben hat man ganz ähnliche concentrisch geschichtete Körper auch in einzelnen Geschwülsten nachgewiesen.

Wenn nun aber Grawitz') sagt, "Corpora amylacea kommen nicht selten in Geschwülsten vor, so ist das doch nicht richtig, wenigstens bei einer etwas scharfen Abgrenzung des Begriffes. Ich habe in der gesammten Literatur nur folgende Fälle finden können, die hier etwa in Frage kommen könnten.

1) E. Wagner (Arch. f. physiol. Heilkunde. 1857) fand bei Krebs des Oesophagus, der Leber u.s. w., in der Peripherie einer krebsigen retroperitonäalen Lymphdrüse zahllose, meist unregelmässig ovale, kuchenförmig platte, mattglänzende Corpora amylacea, von denen einige eine feine concentrische Streifung und alle in oder nahe der Mitte einen oder mehrere Risse hatten. Mit Jodtinctur färbten sie sich hellblan, 10 Stunden später waren sie seamutzig-blan, nach Zusatz von Scbwefelsäure waren sie wieder blau. Diese fanden sich jedoch nur in einem Präparat. A wyloid war in keinem Organ zu finden.

2) Billroth (Beiträge zur pathol. Histologie. 1858) stiess bei seinen Untersucbungen über Lymphdrüsen ebenfalls auf Corpora amylacea. In einer seit 11 Jahren bestehenden Geschwulst am Hals befanden sich einige mit concentrischer Schichtung, andere mit goldgelbem oder dunkelbraunrothem Pigment, welche alle die Jodreaction des A myloids gaben. Ausserdem fand er in tuberculösen Lymphdrüsen concentrisch gescbichtete Concretionen, bei denen jedoch keine Jodreaction gemacht worden ist.

3) Burow (Langenbeck's Archiv. 18) beobachtete dicht oberhalb des Larynx 3 grosse Geschwülste, die nach der Untersuchung Neumann's auf dem Durchschnitt ein graugelbes, homogenes Gewebe von mattem, wachsartigem Glanz darstellten. Mit Jodlösung versetzt; wurde das Gewebe dunkelmahagoniroth, bei Zusatz von Schwefelsäure violett, dunkelblau, braunschwarz, das übrige Gewebe blassgelb. Ferner fanden sich stark glänzende, homogene, rundliche oder unregelmässige glasige Klumpen und Schollen, die durch ihre concentrische Schicbtung an die Prostatamyloide erinnerten. 7 Jahre früher wurden an der gleichen Stelle mehrere Fibroide (nach Neumann's Untersuchung) abgetragen. Er vermutbet deshalb, dass auch die späteren Geschwülste Fibroide waren.

4) Langhans (dieses Archiv. Bd. 38). In einem Carcinom der Lunge fanden sich concentrisch geschichtete Körper, welche sich mit Jod dunkelbraunroth färbten und bei Zusatz von Schwefelsäure nocb

1) Dieses Arebiv. Bd. 94.

Archiv f. pathol. Anat. Bd.140. Hft. 2. 
dunkler in der Farbe wurden, von blauer oder grauer Farbe war nichts zu seben.

5) Steudener (dieses Archiv. Bd. 59) stiess bei der Untersuchung einer Anzahl kleiner Fibrome, die auf der Vorderfläche der Medulla oblongata sassen, auf glänzende, runde und ovale Körperchen, die eine sehr feine concentrische Schichtung aufwiesen. Mit Jod behandelt, nabmen sie nur eine hellgelbe Farbe an, welche aber nacb Zusatz von Schwefelsäure in eine mehr mahagonirothe Farbe überging. Nach einigen Stunden hatten dann die meisten von ihnen eine tiefdunkle schmutzig-blaugrüne Farbe angenommen.

6) Siegert (dieses Archiv. Bd. 129) wies in einem Chondrom der Lunge Corpora amylacea nach mit concentrischer Schichtung, radiärer Streifung und der charakteristischen Jod-und Methylviolettreaction.

Die folgenden 2 Fälle sahen zwar den Corpora amylacea sehr ähnlich, es fehlte ihnen aber die charakteristische Reaction auf Jod und anf Methylviolett.

Zahn (Deutsche Zeitschr. f. Chir. Bd. 22 und dieses Archiv. Bd. 115). In der Wand einer Kiemengangscyste lagen concentrisch geschichtete Körper, die zwar wie Corpora amylacea aussahen, jedoch nicht die charakteristische Jod- und Metbylviolettreaction gaben.

A oyama (dieses Archiv. Bd. 106) fand in einem Mammacarcinom Körper, die den Corpora amylacea ähnlich waren; sie hatten eine concentrische Schichtung und radiäre Streifung, obne jedoch Jod- oder Methylviolettreaction zu geben.

Jeder, der Erfahrung über die Corpora amylacea des normalen Körpers hat, weiss, dass dieselben nicht ganz identisch sind, er weiss, dass z. B. solche des Gehirns sich in einzelnen Dingen anders verhalten, als die der Prostata, ja dass letztere 2 verschiedene Typen darstellen. Eben solche Differenzen findet schon ein flüchtiger Blick auf die oben gegebenen Excerpte über die Corpora amylacea in Geschwülsten heraus. Zunächst sind es ja in allen Fällen homogene, mattglänzende, rundliche Körper mit concentrischer Schichtung. Aber schon die radiäre Streifung fehlt den meisten; nur der Fall von Siegert, A oyama und der meinige hat sie. Und wenn nun gar ausser der concentrischen Schichtung und der radiären Streifung auch noch die typische Reaction auf Jodschwefelsäure, auf Methylviolett zum wesentlichen Inhalt des Begriffs "Corpus amylaceam", gerechnet wird, wie dies Siegert thut und wie dies doch nach der Entstehung des Namens unbedingt gerechtfertigt erscheint, so schrumpft die 
Zahl der Fälle, in denen sich derartige Körper in Geschwïlsten fanden, noch mehr zusammen. Die typische Reaction auf Jod ist ja die, dass die Corpora amylacea sich auf Jodzusatz blau oder grünlich färben, wie dies von Friedreich für die der Lunge, von Virchow für die des Gehirns und von Paulicki für die der Prostata zuerst festgestellt wurde, und dies bei Schwefelsäurezusatz noch klarer wird, während Amyloid sich auf einfachen Jodzusatz braunroth und erst bei Hinzufügen von Schwefelsäure blau oder braunroth färben soll. Diese typische Reaction boten nur die Fälle von Wagner, Siegert und mir, wobei ich betonen muss, dass in meinem Fall häufig auch nur die Amyloidreaction zu Stande kam, wie dies in den Fällen von Billroth, Burow, Steudener, Langhans, stets geschah, während die von Zahn und Aoyama gefundenen Körper gar keine besondere Jodreaction ergaben. Letztere wurden auch durch Methylviolett nicht roth gefärbt. Diese beiden letzteren Fälle müssen wohl trotz der gleichen Morphologie mit aller Bestimmtheit aus dem Kreis der Corpora amylacea ausgeschieden werden.

Es fragt sich aber weiter, ob man blos nach der einzigen differenten Farbenreaction solch bedeutende Unterschiede machen soll, dass man in jenen 5 Fällen nicht von Corpora amylacea spricht, jene Körper also als dem Wesen nach verschiedene Dinge auffasst und nur die Corpora der ersten 3 Fälle als voll gelten lässt. Können es nicht blos verschiedene Stufen eines chemischen Prozesses sein?

Die chemische Zusammensetzung dieser Körper, ihre Stellung zum Amyloid und Hyalin, die eigenthümliche physikalische Beschaffenheit, ihre Entstehungsbedingungen, hat schon seit ihrer Entdeckung das Interesse der Forscher gereizt, ohne dass man zu einem abschliessenden, vollkommen befriedigenden Resultat gekommen wäre. Es herrscht wohl jetzt nach den Untersuchungen von Kekulé und Friedreich ${ }^{1}$, HoppeSeyler ${ }^{2}$, Kühne ${ }^{3}$ ), Schmidt $t^{4}$ u. s. w. kein Zweifel mehr darüber, dass die Corpora amylacea zu den Eiweisskörpern ge-

1) Heidelberger Jahrbuch. 1858. - Dieses Archiv. Bd. 16.

2) Anleitung zur pathol,-chemischen Analyse.

3) Dieses Archiv. Bd.33. - Lehrbuch der physiol. Chemie. 1868.

4) Annalen der Chemie und Pharmacie. CX. 250. 
hören, ebenso wie das Amyloid. Paulicki fand zwar, dass, wenn man Corpora amylacea der Prostata, die sich mit Jod bläuen, mit Speichel behandelt und erwärmt, Gebilde zurückbleiben, die sich durch Jod gar nicht oder nur braunroth, nicht aber blau färben. Er zog daraus den Schluss, dass in diesen Corpora amylacea ein bei Erwärmung in Speichel sich lösender, sich verwandelnder Stoff sei, auf dessen Anwesenheit die Blaufärbung durch Jod beruhe. Ausserdem führte er dieselben durch Speichel in Zucker über, die er durch die Gährungsprobe und die Trommer'sche Probe nachwies. Dabei giebt er zu, dass die Ueberführung in Zucker durchaus nicht in allen Fällen gelingt und dass mit dem weiteren Wachsthum der Amyloidkörper die Stärke allmählich ganz verschwinde. Nun diese Befunde Paulicki's müssen wohl als Thatsachen angenommen werden, wie dies ja auch v. Recklinghausen ${ }^{1}$ ) that, jedoch nur für einzelne Fälle der Prostata. Allgemeine Gültigkeit haben sie nicht, da sie von keiner anderen Seite bestätigt wurden. Ich habe an meinen Präparaten die Probe nachgemacht: ein Theil der Corpora färbt sich nach wie vor bei Jodzusatz bläulich, der andere braunroth. Es geht daraus jedenfalls hervor, dass in meinem Falle die Bläuung nicht auf der Anwesenheit von Stärke beruhen kann, da diese ja durch Speichel in Zucker übergeführt sein müsste. Auch das Ausziehen zerkleinerter Geschwulstmasse mit warmem Wasser und nachfolgender Speichelzusatz brachte keinen Zucker zum Vorschein, der etwa mit der Trommer'schen Probe nachweisbar gewesen wäre. Sie lösen sich überhaupt nicht in Wasser, auch nicht in kochendem, und quellen darin auch nicht auf. Auch das spricht gegen die Stärkenatur. Aber auch die Beobachtung Busk's ${ }^{2}$, dass bei der Betrachtung der Corpora im polarisirten Licht ein Kreuz zu sehen sei, gelang es mir nicht, ebenso wie Virchow und Donders, zu bestätigen. Also auch dieser Beweis für die Stärke in den Corpora amylacea ist in meinem Fall nicht vorhanden. Etwas Sicheres und Genaues ist aber über die chemische Zusammensetzung nicht bekannt; complicirt wird die Sache noch dadurch, dass die Corpora ja nicht einmal

1) Handbuch der allgemeinen Pathologie u. s. w.

2) $1 . \mathrm{c}$. 
einen absolut einheitlichen, chemischen Körper darstellen, färben sich doch oft in demselben Schnitte die einen mit Jod braunroth, die anderen bläulich; ja sogar an einem und demselben Körper sah man verschiedenfarbige, blave oder braunrothe Ringe, wie Paulicki schon angiebt und wie ich es ebenfalls in meinen Präparaten sehen konnte. Die Analogie aus dem Pflanzenreich, die Paulicki einst heranzog, zur Erklärung für dieses Factum, nehmlich die wechselnde Zusammensetzung der Stärkekörner aus Stärke und Cellulose und ihre dementsprechende verschiedene Farbenreaction, kann eben doch nur als eine Analogie gelten, nicht als Erklärung. Es ist im Ganzen doch sehr unwahrscheinlich, dass in den bla ugefärbten Corpora 2 wesentlich verschiedene Stoffe, ein braunroth und ein blau sich färbender, vorhanden sind, die nicht in einander übergingen, in den braugrothen aber nur der erstere; sondern es liegt doch wohl näher, dass es nur je ein Stoff ist, die etwas different sind, die sich einer in den anderen umwandeln können, so dass sie nur verschiedene Stufen eines Prozesses bedeuten. Da nun jene, oben erwähnte, von der typischen abweichende Jodreaction mancher Corpora amylacea, mancher Theile derselben sich mit der Reaction des Amyloids deckt, so kommen wir damit zu der Frage, in welchem Verhältniss stehen die Corpora amylacea, speciell uuserer Geschwulst zu dem Amyloid und ferner zu dem Hyalin, jenem anderen so ähnlichen Stoffe. Letzteres wird ja ziemlich häufig in Geschwülsten beobachtet. Aber es sind auch eine Reihe Fälle bekannt von Amyloidgeschwülsten, von sogenanntem localem Amyloid, so an den Augenlidern von Rählmann' ${ }^{1}$ ), Leber ${ }^{2}$ ), Reymond ${ }^{3}$ ), Oettingen ${ }^{4}$ ), Hippel ${ }^{5}$ ), Kraus ${ }^{6}$ ) und Anderen, an der Zunge von $\mathrm{Kraus}^{7}$ ), $\mathrm{Zahn}^{8}$ ), Ziegler ${ }^{9}$ ), in

1) Archiv f. Augenbeilkunde. X. - Dieses Archiv. Bd. 87.

$\left.{ }^{2}\right)$ Archiv f. Ophthalmologie. XIX.

3) Annali di Ottalmologia. IV.

4) Dorpater med. Zeitschr. II. 1871 .

5) Archiv f. Ophthalmologie. XXV.

6) Zeitschr. f. Heilkunde. 1885.

7) Zeitschr. f. Heilkunde. 1885.

8) Deutsche Zeitschr. f. Chir. Bd. 22.

9) Dieses Archiv. Bd. 65. 
der Trachea von $\operatorname{Kraus}^{1}$ ), Balser ${ }^{2}$ ), in der Nase bei Thieren von Grawitz ${ }^{3}$, Rabe ${ }^{4}$ ), in einem Enchondroma mixtum von Lesser ${ }^{5}$ ), in einer Ekchondrosis von Virchow ${ }^{6}$ ), in Strumen von Friedreich ${ }^{7}$ ), Beckmann ${ }^{8}$ ), Virchow ${ }^{9}$ ), Laycock ${ }^{10}$ ). Einzelne dieser Autoren sahen auch in denselben Geschwülsten neben dem Amyloid Hyalin, wie z. B. Rählmann und Zahn.

Die Autoren denken über die Beziehungen dieser Stoffe zu einander sehr verschieden. Virchow ${ }^{11}$ ) machte anfänglich keine Unterscheidung $z$ wischen Corpora amylacea und Amyloid, er rechnete erstere unter das Amyloid. Stilling ${ }^{12}$ ) sagte über die Corpora amylacea der Prostata, sie wären genetisch nicht verschieden von Hyalin und Amyloid, $Z a h n^{13}$ ) und Rählmann ${ }^{14}$ ) nehmen Hyalin als die Vorstufe des Amyloid. Posner ${ }^{15}$ ) dagegen trennt die Corpora amylacea der Prostata vom Amyloid $a b$ auf Grund der verschiedenen Färbung durch Jod und sonstiger chemischer Reactionen, ebenso schied auch Recklinghausen ${ }^{16}$ ) die Corpora amylacea der Lunge, der Prostata und des Centralnervensystems rom Amyloid und $\mathrm{Ceci}^{17}$ ) die des Centralnervensystems, deren Osmiumsäurereaction beim Amyloid nicht eintritt. Eberth ${ }^{18}$ ) erklärt bestimmt, dass die Corpora amylacea nichts mit Amyloid zu thun hätten. Auch Wich-

1) Zeitschr. f. Heilkunde. 1886.

2) Dieses Archiv. Bd. 91.

3) Dieses Arehiv. Bd. 94.

4) Jahresbericht der Königl. Arzueischule. Hannover 1884-85.

5) Dieses Archiv. Bd. 69.

6) Virchow, Die Geschwülste. Bd. I.

7 Dieses Arehiv. Bd. 11.

8) Dieses Archiv. Bd. 13.

9) Virchow, Die Geschwülste. Bd. III.

19) Edinb. med. Journ. 1863.

11) Dieses Archiv. Bd. 8.

12) a. a. 0.

13) a. a. 0 .

14) a. a. 0 .

15) a. a. 0.

16) Handbuch d. allg. Patbol. des Kreislaufs. 1883. S. 398.

17) $1 . \mathrm{c}$.

18) Mikroskop. Technik. 
mann ${ }^{1}$ ), ebenso Síegert' ${ }^{2}$, als letzter Autor über diese Frage, trennt dieselben scharf vom Amyloid. Während also die einen die Corpora amylacea streng vom Amyloid scheiden, halten andere sie für verwandt.

Das Verhältniss des Amyloids zum Hyalin dagegen fassen die meisten im Sinne Rählmann's und Recklinghausen's auf, welch letzterer, S. 417 seines Handbuches, sich so darüber ausspricht: Eine scharfe Grenze zwischen dem Amyloid und dem Hyalin lässt sich, wie die bisherige Betrachtung zeigt, nicht ziehen. Nicht nur theilen beide Substanzen viele morphologische Eigenschaften, sondern kommen auch neben einander vor, wie Rählmann in einem Falle von Amyloidtumor der Conjunctiva constatirte. Ausserdem sind die Reactionen des Amyloids, wie des Hyalins in einem gewissen Grade variabel. Alle diese Momente weisen wohl daraufhin, dass das Hyalin und das Amyloid nur verschiedene Stufen einer gleichartigen Umwandlung der Gewebselemente repräsentiren $u$. s. w.

Nun muss es aber doch auffallen, dass in meinen Präparaten 1) rundliche Körper vorkommen, die in Allem den Corpora amylacea entsprechen, 2) unregelmässig geformte, zum Theil sehr grosse Schollen sich finden, die dem Aussehen nach den amyloiden vollständig gleichen und dieselben Reactionen geben, wie jene Corpora amylacea. Man müsste dann also annehmen, dass die specifische nicht amyloide Substanz der Corpora amylacea, auch wie die amyloide in Schollenform vorkäme. Der Fall Ziegler's von Osteofibrom der Zunge dürfte dann nicht zum Amyloid gerechnet werden, da die glänzenden Schollen auf Jodzusatz sich bläuten, also die Färbung der Corpora amylacea annahmen, ohne jedoch Form und Struktur derselben zu haben. Eine andere Möglichkeit wäre die, es käme in meinem Falle beides, Corpora amylacea und Amyloid, neben einander vor, ohne mit einander irgend etwas zu thun zu haben; dann sähe man keinen Grund ein, sie, ihrer chemischen Zusammensetzung nach, als etwas Verschiedenes zu betrachten, da die Reactionen alle die gleichen sind; oder schliesslich, die Corpora amylacea sind eben auch nichts weiter als Amyloid, jedoch besonders geformt. Für

1) Ziegler's Beiträge z. path. Anat. XIII. 1893.

2) Dieses Archiv. Bd. 129. 
die erste oder dritte dieser Annahmen spricht doch sehr, dass sämmtliche mikrochemische Reactionen der Corpora amylacea und jener Schollen identisch sind. Ausserdem aber ist die Jodreaction durchaus nicht bei allen Corpora amylacea und beim Amyloid so präcis. Wie schon oben erwähnt, fand ich bei Jodzusatz neben bläulichen, bläulich-grünen Körpern vielfach rothbraune, von im Uebrigen gleicher Struktur und Reaction; ebenso verhielten sich die Schollen. Soll man nun etwa sagen, jene rothbraunen Körper sind keine Corpora amylacea, sondern Amyloid und jene sich bläuenden Schollen und Gefässmäntel sind kein Amyloid, sondern Corpora-amylacea-ähnliche Substanz? Soll man die verschiedenen concentrischen Ringe um die Körper, die sich der eine roth, der andere bläulich färbten, verschieden als amyloid oder amylacisch betrachten? Gewiss nicht. Die Jodreaction ist eben nicht so präcis, sie ist leicht variabel, wie dies ja auch schon aus den sehr verschiedenen Farbennuancen hervorgeht, die man in ein und demselben Schnitte bekommt. In dem oben angeführten Citat von Recklinghausen ist ja diese Ansicht auch schon zum Ausdruck gekommen. Uebrigens wird dies auch in allen Büchern über mikroskopische Technik angegeben. Freilich ist andererseits von Posner noch auf gewisse andere chemische Unterschiede zwischen den Corpora amylacea der Prostata und dem Amyloid aufmerksam gemacht worden, auf Grund deren er beide Substanzen trennt. Kochender Alkoholäther löst nach Fürbringer die Corpora amylacea der Prostata leicht bis auf unscheinbare Reste, Amyloid nicht. Amyloid wird durch kochende concentrirte Salpetersäure zerstört, die Corpora amylacea der Prostata nicht, sie zeigen noch schöne Reaction. Aber gerade diese Reactionen Posner's sind in meinem Falle nicht geeignet, eine Abtrennung der Corpora amylacea von dem Amyloid zu motiviren, da sich die Corpora amylacea ganz ebenso verhielten, wie die anderen Schollen. Sie entscheiden aber die Frage, ob die Substanz, aus der jene beiden bestehen, eine amyloide ist oder eine andere specifische, denn für beide Proben tritt nur die Reaction des Amyloids ein: durch kochende concentrirte Salpetersäure werden sie ganz gelöst, durch kochenden Aetheralkohol aber nicht, so dass sie nach dieser Behandlung ihre gewöhnliche Jodreaction noch geben. 
Nun sagt Virchow schon im 8 . Bande seines Archivs, dass die Corpora amylacea in heissem Alkoholäther nicht löslich sind, dass concentrirte Säuren und Alkalien sie sofort zerstörten, hat also bei seinen Präparaten dasselbe gefunden, während ich freilich den anderen Befund nicht erheben konnte, dass die Corpora in kochendem Wasser aufquellen und sich lösen wie die pflanzliche Stärke. Ich komme daher zu dem Schluss, dass wenigstens in meinem Falle kein Grund vorhanden ist, die Corpora amylacea, was ihre chemische Zusammensetzung anbetrifft, von jene Schollen zu trennen, und ich glaube ferner, dass sie ebenso wie diese aus amyloider Substanz bestehen, da ich keine Reaction finde, die beim Amyloid anders wäre als bei ihnen, ist ja doch die Bläunng des Amyloids durch einfachen Jodzusatz im Ziegler'schen Falle auch schon beobachtet.

Dass Glykogen ganz aus dem spiele bleibt, das geht wohl aus dem Verhalten der Corpora amylacea u. s. w. gegenüber Wasser, Speichel, Jod u. s. w. zur Genüge hervor. - Ob nun aber ein weiterer Zusammenhang zwischen diesem Amyloid und dem Hyalin besteht, wie dies Stilling, Rählmann annimmt, dafür kann ich in meinen Befunden keinen Anhaltspunkt finden. Eine Prüfung meiner Präparate, nach der Methode von Ernst mit van Gieson'schem Gemisch ergab niemals Rothfärbung der Corpora, wie dies beim ächten Hyalin der Fall sein soll, wenn ihr Farbenton auch ab and zu mehr dem Roth sich zuneigte, als der des Zellprotoplasmas.

Wenn wir nun die Annahme machen, dass es sich hier um Bildung von Amyloid handelt, so müssen wir, da dies gewöhnlich nicht in der Form von Corpora amylacea vorkommt, uns weiter fragen, wodurch können sich aus der gewöhnlich strukturlosen Form der amyloiden Massen jene concentrisch geschichteten, radiär gestreiften Körper bilden? Damit taucht aber als nothwendigerweise vorber $\mathrm{zu}$ erledigend die Frage auf, wie und wo bildet sich überhaupt das Amyloid. Ist es eine einfache Umw̧andlung gewisser Zellen, wie z. B. die colloide Umwandlung der Schilddrüsenzellen, entsteht das Amyloid schon in der Zelle, oder erst ausserhalb, in welchen Geweben spielt sich diese Veränderung überhaupt $a b$ ? Die Differenzen, die über den Ort der amyloiden Degeneration, über das betroffene Gewebe bis 
vor Kurzem bestanden, sind wohl beigelegt, die Meinungen haben sich geklärt und es herrscht jetzt wohl kaum ein $Z_{\text {weifel }}$ mehr darüber, dass Eberth $^{1}$ ) mit seinen Anschaungen Recht hat, dass im Allgemeinen die amyloide Degeneration hauptsächlich nur das Bindegewebe betrifft, speciell das Bindegewebe um die Gefässe herum, dass dagegen die Abkömmlinge des Ektoderm und Entoderm freibleiben. Freilich über die Nichtbetheiligung der Leberzellen sind sich noch nicht alle Autoren einig. Für die Lymphdrüsen und die Milz speciell vertritt Eberth den Standpunkt, dass die Lymphzellen nicht betheiligt. sind, dass diese nur durch das amyloid gequollene Reticulum erdrückt werden. Auf diese Lymphzellen aber gerade führte Virchow ${ }^{2}$ ) die Entwickelung der Corpora amylacea zurück, die ja von Virchow mit dem Amyloid als gleichwerthig betrachtet und deshalb wohl auch von den früheren Autoren oft als Corpora amyloidea bezeichnet wurden. Virchow meinte, in den Lymphdrüsen gingen sie nicht aus einer, sondern aus mehreren Zellen hervor, einer ganzen Gruppe. Was in der Literatur angegeben ist, das weist auf eine solche Entstehung aus den Zellen.

Meine Präparate sprechen nun sehr dafür, dass in meinem Falle ausser dem $Z$ wischenbindegewebe noch die Geschwulstzellen stark betheiligt sind an der Bildung des Amyloids. Es scheint mir kein Zweifel zu sein, dass die eigentlichen Geschwulstzellen, die Sarcomrundzellen, es sind, die in meinem Falle der Sitz der Umwandlung waren. Man sieht ja zwar homogene Balken und Züge, die amyloid entartetem Bindegewebe entsprechen, man sieht $a b$ und zu amyloide Ringe um die Gefässe herum, aber die Corpora amylacea, die Schollen sind aus den Sarcomzellen entstanden. Das zarte Maschennetz um die Corpora, das sehr deutlich erhalten ist und sich deutlich gegen die Corpora amylacea abhebt, beweist dies. Nach meinen Präparaten möchte ich annehmen, dass die Corpora in der Weise sich bilden, dass das Protoplasma der Zellen zum Theil eine chemische Umwandlung erleidet, so dass Vacuolen entstehen, dass dann weiter diese homogenen Kugeln aus der Zelle aus-

1) Dieses Archiv. Bd. 80.

2) Dieses Arehiv. Bd. 8 . 
treten oder dass die Zellen ganz aufquellen, homogen, glasig werden und der Zellkern zu Grunde geht; dabei werden sie ganz natürlich rund. Nun liegt diese Kugel inmitten anderer Zellen als Centrum; die an die homogene Kugel anstossenden Zellen verändern sich ebenso, die flüssigen oder jedenfalls weichen Massen vereinigen sich und bilden nun einen Ring um jene erste Kugel, mit der sie sich zwar vereinigen, jedoch so, dass die Vereinigungslinie sichtbar bleibt. Wenn dies nun weiter geht, so entstehen mehrere solche Ringe um die primäre Kugel. Die Maschensepten werden gedehnt, gehen wohl auch, wenigstens die feineren, zarteren, zu Grunde und die noch nicht veränderten Zellen werden bei Seite gedrängt. Man sieht sie vielfach den Corpora angeschmiegt ansitzen. So würde sich wohl die concentrische Schichtung erklären lassen. Zweifellos können nun mehrere solche Körper sich vereinigen und dann grosse Schollen bilden. Dafür habe ich ganz beweisende Stellen gefunden. An den Berührungs-, Verschmelzungsflächen gehen sie in einander über, da verliert sich die concentrische Schichtung; so wird es möglich, dass aus einem solchen Conglomerat von selbständigen Kugeln eine grosse Scholle wird mit einheitlicher concentrischer Schichtung. An manchen Stellen kann man ja eben noch die einzelnen Corpora erkennen, die schon zusammengeflossen sind, oft deuten noch die Reste, die Kerne der Maschen, die Grenzen der einzelnen Corpora an. Auch an der radiären Streifung kann man das sehr gut erkennen; an den Stellen, wo die Vereinigung ist, hört die radiäre Streifung auf, wie aus Fig. 3 ersichtlich ist; an der Grenze geht sie in die des anliegenden Körpers über. Zweifellos geht aber daraus hervor, dass die Corpora im Leben eine relativ weiche, modellirbare Substanz sein müsse, sonst würden sie sich doch nicht vereinigen können, namentlich in der Weise, dass die Schichtungen in einander übergehen, ja, dass auch die radiäre Streifung sich danach formirt. Daher sind sie wohl auch meist rund; die Bindegewebsmasche wird gedehnt und übt dabei einen gleichmässigen Druck auf den in ihr gelegenen formbaren Klumpen. Ich vermuthe, dass gerade deshalb, weil in meinem Fall die Geschwulstzellen in einem Maschennetz lagen, das formirend einwirken konnte, bei der Umwandlung nicht unregelmässige Klumpen amyloider Masse 
resultirten, wie in den Fällen von localem Amyloid, die bis jetzt beschrieben sind, sondern runde Kugeln. Als Stütze für diese Vermuthung kann auch die Thatsache gelten, dass in den wenigen Fällen, wo Amyloid sich in Lymphdrüsen fand, es auch runde Form hatte.

Dass die concentrischen Ringe zu verschiedenen Zeiten entstanden sind, dafür nehme ich als weiteren Beweis, ihre oft verschiedene Färbbarkeit mit Jod, die ich schon erwähnte. Diese deutet vielleicht darauf, dass das Amyloid nicht immer und zu allen Zeiten die ganz gleiche chemische Beschaffenheit hat. Es spricht ferner für diesen Enstehungsmodus, dass die kleinen Corpora in meinen Präparaten nie concentrisch geschichtet waren, sie entsprachen der ziemlich grossen, ungeschichteten Kugel, welche den Kern der grösseren Corpora amylacea bildete. Man wird mir nun vielleicht einwerfen, dass ich noch keinen Beweis beigebracht hätte, dafür, dass wirklich die Geschwulstzelle sich so umwandle, dass wirklich der erste Anfang in den runden Zellen gelegen sei, Die Beweise liegen erstens darin, dass ich verschieden grosse Zellen sah mit homogenen Kugeln im Innern, wenn dies auch etwas undeutlich zu sehen war und diese Zellen selten waren. Auch in Riesenzellen nahm ich das wahr; man sah neben der kernlosen homogenen Kugel eine Anzahl von Zellkernen liegen; meist waren aber die Riesenzellen ohne solche Veränderungen. Bei Jodfärbung jedoch habe ich diese Dinge nicht beobachtet, da wurden die Zellen doch nicht so präcis, die Abgrenzung wurde nicht so deutlich, um einigermaassen sicher zu sein, dass die Kugeln wirklich in den Zellen lagen, nicht blos ihnen anlagen. Deshalb habe ich auch nicht mit Sicherheit constatiren können, ob die Kugeln in den Zellen wirkliches Amyloid waren. Es ist ja aber auch noch lange nicht gesagt, dass diese Veränderung in den Zellen gleich eine amyloide ist, und es wäre sehr wohl denkbar, dass diese erst einträte, sobald jene Kugel aus der Zelle herausgetreten ist, bezw. sobald die ganze Zelle sich umgewandelt hat und der Kern zu Grunde gegangen ist, unter Mitwirkung des circulirenden Eiweisses, welches letztere nach Ziegler ${ }^{1}$ ) hauptsächlich das Material dazu

1) Lehrbuch der. allg. pathol. Anatomie. 
liefert. $O b$ bei jenem Vorgang die Zelle direct zu Grunde geht, in der Weise, dass das ganze Protoplasma sich umwandelt und der Kern verschwindet, oder ob sich nur ein Theil umwandelt, der dann als homogene Kugel quasi ausgestossen wird, während die nun sehr verkleinerte Zelle durch die wachsenden Amyloidkörper zusammengedrückt wird und erst später in Folge dessen zu Grunde geht, dafür habe ich keine entscheidenden Bilder gewinnen können, doch deuten meine Beobachtungen mehr auf diesen letzten Modus hin.

Noch eine Beobachtung an den Corpora amylacea bleibt mir zu erklären übrig, das ist die radiäre Streifung. Nur einzelne Autoren machen den Versuch, die übrigen schweigen über diesen Punkt. Aus dem Vergleich der Corpora amylacea der Prostata mit den Blasensteinen, denen ja auch die Corpora meiner Präparate stellenweise ganz ausserordentlich ähneln, erwuchs Posner die Auffassung, dass die radiären Streifen hervorgerufen würden, durch die Einlagerung von Krystallen, und zwar von Lecithinkrystallen. Wenn bei Blasensteinen eine radiäre Streifung vorhanden ist, so beruht sie darauf, dass ein organisches Gerüst mit Radien besteht, zwischen welche die Steinablagerung stattfindet. Auch in der Steinwelt der Mineralogie beruht die radiäre Streifung gewöhnlich auf der eigenthümlichen Anordnung der Krystalle, so dass dann eine sowohl concentrische als radiäre Streifung der Gesteine resultirt. $O b$ in meinem Präparate auch diese Ursache vorliegt, kann ich nicht sagen, da dasselbe schon seit einer Reihe von Jahren in Alkohol gelegen hat und Lecithin ja in diesem leicht löslich ist. Nach Auflösung der Krystalle können natürlich ihre Lagerstätten als radiäre Streifen sichtbar bleiben. An einzelnen Corpora sieht die radiäre Streifung ja so aus, als ob ein radienartig angeordneter Büschel von Krystallen da läge. Bei der Mehrzahl aber erscheint dies unwahrscheinlich. Faltungen, Schrumpfungen können es nicht gut sein, dagegen sprechen die Bilder der grösseren, aus mehreren Corpora hervorgegangenen Schollen und die Art und Weise, wie die radiäre Streifung bei diesen Schollen in einander übergeht und an den Verschmelzungsflächen schwindet. Die Regelmässigkeit dieser Streifen, ihr Auftreten in der Peripherie der grossen Schollen lässt auch die Vermuthung nicht 
zur Geltung kommen, dass die Streifen etwa etwas dichteren Protoplasmafäden im Zellinnern entsprächen, die durch die Ausdehnung der Zelle glatt gestreckt worden wären. Zu einer bestimmten, mich selbst überzeugenden Deutung für diesen Befund der radiären Streifung bin ich nicht gelangt.

Das Resumé lautet also so: Es handelt sich in unserem Fall um ein endostales Rund- und Riesenzellensarcom des Sternum mit amyloider Entartung. Die letztere wurde sowohl in der Form des gewöhnlichen localen Amyloids als der typischen Corpora amylacea beobachtet. Letztere werden also in diesem Falle, was ihre chemische Constitution anbetrifft dem Amyloid gleichgesetzt. Es ist das meines Wissens der einzige Knochentumor, in welchem eine derartige Beobachtung gemacht wurde, überhaupt der einzige Fall, dass Amyloid in einem Knochen beobachtet wurde. Nur bei Rokitansky') findet sich die Notiz, dass er wiederholt Corpora amylacea in osteomalacischem Knochen angetroffen habe. Man kann aber aus seiner diesbezüglichen Bemerkung nicht ersehen, ob er auch bei diesen von ihm beobachteten Fällen die charakteristische Färbung erhalten hat. Deshalb sind diese Fälle aus den früher erwähnten Gründen nicht mitzurechnen. - Von amyloider Erkrankung anderer Organe wurde bei unserer Patientin nichts beobachtet. Doch will ich nicht unerwähnt lassen, dass Patientin $1 \frac{1}{2}$ Jahre nach der Operation starb, nachdem sie einen Monate lang dauernden Rheumatismus durchgemacht hatte, und dass sie eine Zeit lang vor dem Tode an Albuminurie litt.

Ausser der im Text citirten Literatur wurde benutzt:

Kyber, Studien über die amyloide Degeneration. Dorpat 1871. - Dieses Archiv. Bd. 81.

Eberth, Dieses Archiv. Bd. 84 .

Bötticher, Dieses Archiv. Bd. 72, 84.

Curschmann, Dieses Archiv. Bd. 79.

Burchardt, Dieses Archiv. Bd. 117.

Zahn, Dieses Archiv. Bd. 115.

Besser, Dieses Archiv. Bd. 36.

Mayer, Dieses Archiv. Bd. 19.

1) Lehrbuch der pathol. Anatomie. 
Slavjansky, Dieses Archiv. Bd.51. Virchow's Cellularpathologie.

Klebs, Archiv f. experimentelle Pathol. u. s. w. 1879. Bd.X.

\section{Erklärung der Abbildungen. Tafel VII.}

Fig. 1. Grosse Scholle mit peripherischer radiärer Streifung, mehrere concentrisch geschichtete Corpora enthaltend.

Fig. 2. Mehrere Corpora amylacea mit dicht angelagerten, nicht mebr ganz deutlich abgegrenzten Zellen.

Fig. 3. Scholle mit peripherischer radiärer Streifung, wohl aus 3 Corpora zusammengeflossen.

Fig. 4. Eigenthümlich geformte Scholle mit mehreren Zonen und durchgehender radiärer Streifung.

Fig. 5. Riesenzelle mit diffus vertheilten Kernen.

Fig. 6. Riesenzelle mit peripherischen Kernen und leicht granulirtem Protoplasma.

Fig. 7, 8, 9. Verschieden grosse Zellen mit bellen, nicht sehr deutlichen Vacuolen.

Fig. 10. Corpus amylaceum, radiär gestreift, mit dicht ansitzenden, grossen Zellen, in welche hinein die radiäre Streifung sich erstreckt.

Fig. 11. Aufgequollene Zelle mit peripherisch gestelltem Kern.

Fig. 12. Amyloider Mantel um ein Gefässlumen, dem Endothelrohr dicht ansitzend.

Fig. 13. Amyloider Mantel um ein Gefässlumen, von dem Endothelrohr durch lockeres Bindegewebe getrennt. 

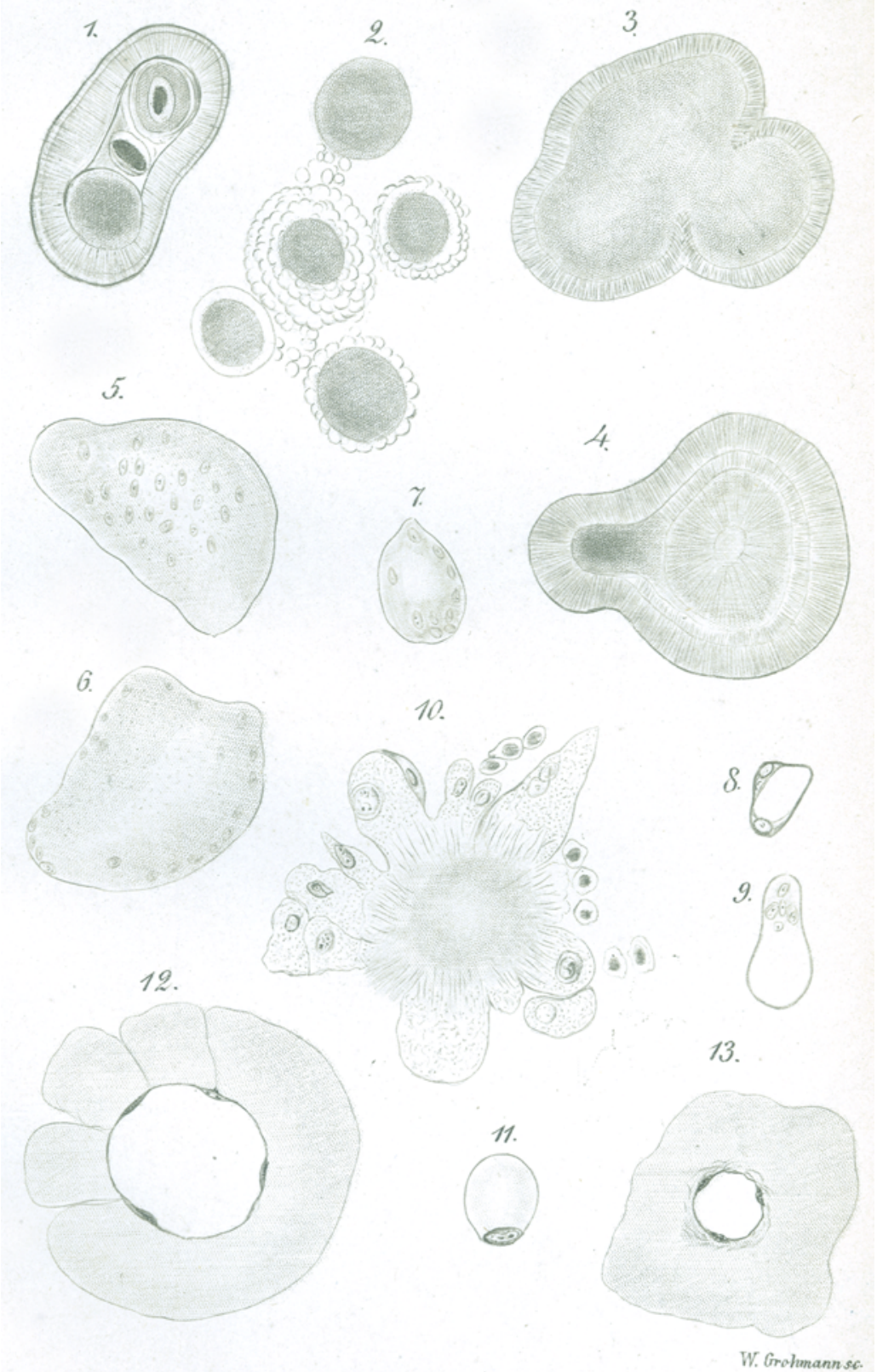